\title{
Current Ablation Strategies for Persistent and Long-Standing Persistent Atrial Fibrillation
}

\author{
Konstantinos P. Letsas, Michael Efremidis, Charalampos Charalampous, Spyros Tsikrikas, \\ and Antonios Sideris
}

Laboratory of Invasive Cardiac Electrophysiology, Evangelismos General Hospital of Athens, 10676 Athens, Greece

Correspondence should be addressed to Konstantinos P. Letsas, k.letsas@mail.gr

Received 29 October 2010; Revised 7 December 2010; Accepted 10 January 2011

Academic Editor: Adrian Baranchuk

Copyright $\odot 2011$ Konstantinos P. Letsas et al. This is an open access article distributed under the Creative Commons Attribution License, which permits unrestricted use, distribution, and reproduction in any medium, provided the original work is properly cited.

\begin{abstract}
Atrial fibrillation (AF) is associated with an increased risk of cardiac and overall mortality. Restoration and maintenance of sinus rhythm is of paramount importance if it can be accomplished without the use of antiarrhythmic drugs. Catheter ablation has evolved into a well-established treatment option for patients with symptomatic, drug-refractory AF. Ablation strategies which target the pulmonary veins are the cornerstone of AF ablation procedures, irrespective of the AF type. Ablation strategies in the setting of persistent and long-standing persistent AF are more complex. Many centers follow a stepwise ablation approach including pulmonary vein antral isolation as the initial step, electrogram-based ablation at sites exhibiting complex fractionated atrial electrograms, and linear lesions. Up to now, no single strategy is uniformly effective in patients with persistent and longstanding persistent AF. The present study reviewed the efficacy of the current ablation strategies for persistent and long-standing persistent $\mathrm{AF}$.
\end{abstract}

\section{Introduction}

Atrial fibrillation (AF) is associated with a 2-fold risk of cardiac and overall mortality [1]. Restoration and maintenance of sinus rhythm is of paramount importance if it can be accomplished without the use of antiarrhythmic drugs [2]. Catheter ablation of AF has been widely accepted as an important therapeutic modality for the treatment of patients with symptomatic AF, refractory or intolerant to at least one class I or III antiarrhythmic medication [3-5]. Previous studies have clearly demonstrated the superiority of catheter ablation over antiarrhythmic drug treatment, even in patients with persistent AF [6-11]. The recently published ESC guidelines state that ablation of persistent symptomatic AF that is refractory to antiarrhythmic therapy should be considered a treatment option (class IIa, level of evidence B) [5]. In addition, catheter ablation of AF may also be considered in patients with symptomatic long-standing persistent AF refractory to antiarrhythmic drugs (class IIb, level of evidence C) [5].
Following the work of Haïssaguerre et al. [12], catheter ablation of paroxysmal AF aiming at electrical pulmonary vein isolation (PVI) results in maintenance of sinus rhythm in 60 to $85 \%$ of patients $[13,14]$. On the contrary, PVI is considered insufficient to eliminate persistent or long-standing persistent AF (more that one year) leading to significantly lower success rate of this method $[3,4,15,16]$. Substrate modification is usually required in the setting of persistent and long-standing persistent AF $[15,16]$. Although different ablation strategies have been reported in persistent and long-standing persistent AF, the reproducibility of these techniques is considered inconsistent. This paper highlights on the current catheter ablation strategies for persistent and long-standing persistent AF.

\section{Pathophysiology of Atrial Fibrillation}

The pathophysiology of AF is multifactorial, complex, and not well defined. Up to date, two main theories have been reported for the initiation and maintenance of AF. The 


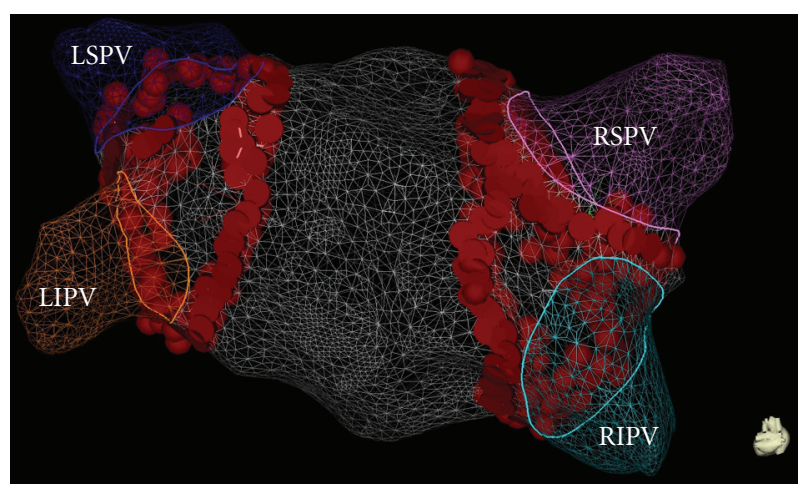

Figure 1: Three-dimensional reconstruction of the left atrium using the CARTO 3 map system (Biosence Webster, USA) showing large circumferential ablation lesions around both ipsilateral veins. In this example, additional radiofrequency energy was applied on the interpulmonary isthmus following the large circumferential lesion creating a "figure of eight" model. LSPV: left superior pulmonary vein; LIPV: left inferior pulmonary vein; RSPV: right superior pulmonary vein; RIPV: right inferior pulmonary vein.

single-focus hypothesis advocates that $\mathrm{AF}$ is due to a single automatic focus or a microreentrant circuit $[5,17]$. Triggers that originate from the PVs and other thoracic veins appear to be the primary mechanism of AF, particularly in subjects with paroxysmal AF $[5,12,18]$. The multiple re-entrant wavelet hypothesis supports that fractionation of wavefronts propagating through the atria results in self-perpetuating "daughter wavelets" [19, 20]. Multiple re-entrant wavelets are separated by lines of functional conduction block, and AF is perpetuated by slowed conduction, increased atrial mass, and shorter refractory periods $[5,19,20]$. The relationship between these mechanisms is complex, and they often coexist in the same patient, particularly in the setting of persistent AF or long-standing persistent AF [21]. Autonomic factors have also been implicated in the generation of complex fractionated atrial electrograms (CFAEs), an important substrate of $\mathrm{AF}[3,4]$. Electroanatomic remodeling that develops as AF progresses from paroxysmal to persistent and permanent has been well demonstrated to further facilitate $\mathrm{AF}$ ("AF begets $A F$ ") [22]. Atrial dilation, interstitial fibrosis, uncoupling of the myofibrils, loss of myofibrils, deposition of extracellular matrix, loss of gap junctions, resultant anisotropy, conduction slowing and/or block, and shortening of the effective refractory period may facilitate reentry, which is critical to perpetuation of AF [3]. As a result of progressive electroanatomic remodelling, mechanisms other than PV arrhythmogenicity are strongly involved and perpetuate AF. Therefore, in addition to PVI, left atrial ablation that modifies the substrate is required in the setting of persistent and long-standing persistent AF.

\section{Ablation Strategies and Success Rates in Persistent AF}

3.1. Pulmonary Vein Antral Isolation. Ablation strategies which target the PV ostium or the PV antrum aiming at electrical disconnection of the PVs are the cornerstone of AF ablation procedures. Initial attempts targeted the arrhythmogenic activity within the PVs using a focal approach [12]. Due to the high risk of PV stenosis and the high rate of recurrence, complete electrical isolation of the PVs by segmental ostial ablation quickly replaced the initial approach $[23,24]$. Successful electrical isolation is defined by loss of PV potentials (entrance block) and failure to capture left atrium during pacing from the PV (exit block) (Figure 1). Pappone et al. have introduced the circumferential PV ablation (PV antral ablation) without electrical PV disconnection [25]. This technique involves applications of radiofrequency energy $1-2 \mathrm{~cm}$ away from the ostia of the PVs until the local electrogram amplitude decreased by $\geq 80 \%$ or to $<0.1 \mathrm{mV}$. In a randomized trial, Arentz et al. have demonstrated that isolation of a large circumferential area around both ipsilateral PVs with verification of conduction block (pulmonary vein antral isolation (PVAI)) is a more effective treatment of AF than isolation of each individual PV using a segmental approach [26]. The end point of ablation is the absence or dissociation of residual PV potentials in the isolated area as documented with the circular mapping catheter. Figure 2 shows the threedimensional reconstruction of the left atrium with large circumferential ablation lesions around both ipsilateral veins and additional ablation lines on the interpulmonary isthmus creating a "figure of eight" model.

Segmental PVI or PVAI confirmed by absence or dissociation of $\mathrm{PV}$ potentials is the most effective strategy for treatment of most patients with paroxysmal AF [2729]. Despite achieving a very high rate of electrical PVI, PVI strategies alone have consistently demonstrated a lower success rate in patients with persistent and long-standing persistent AF compared to paroxysmal AF [3, 4, 29-32]. $\mathrm{PVI}$ alone in persistent and long-standing persistent AF was associated with a single-procedure, drug-free success rate ranging from $21 \%$ to $22 \%$ at almost 2 years [33]. On the contrary, PVAI display higher success rates. In a randomized trial, Oral et al. have shown that the success rate of the index procedure after successful PVAI was $36 \%$ in patients with long-standing persistent AF [34]. In the same line, Elayi et al. reported a $40 \%$ success rate following PVAI in patients with long-standing persistent AF [35]. In a recent study, Tilz et al. have shown that PVAI is sufficient to restore SR in $43.2 \%$ of patients with long-standing persistent AF [36]. Cheema et al. have demonstrated that single-procedure success rate is higher in patients who were in $\mathrm{AF}$ for $\leq 1$ year compared with those in AF for $>1$ year (50\% versus 20\%, resp.) [37]. The variations in success rates of these studies may be attributed to the different definitions of long-standing $\mathrm{AF}$ as well as at the different end-points for AF ablation. Nevertheless, PVAI with electrical disconnection of the encircled veins within the ablated margins has become the preferable ablation strategy in patients with persistent and long-standing persistent AF. It is of major importance to perform a continuous circumferential line around both ispilateral PVs, and then to validate for electrical PV disconnection aiming at the earliest residual potential recorded by the circular mapping catheter. 


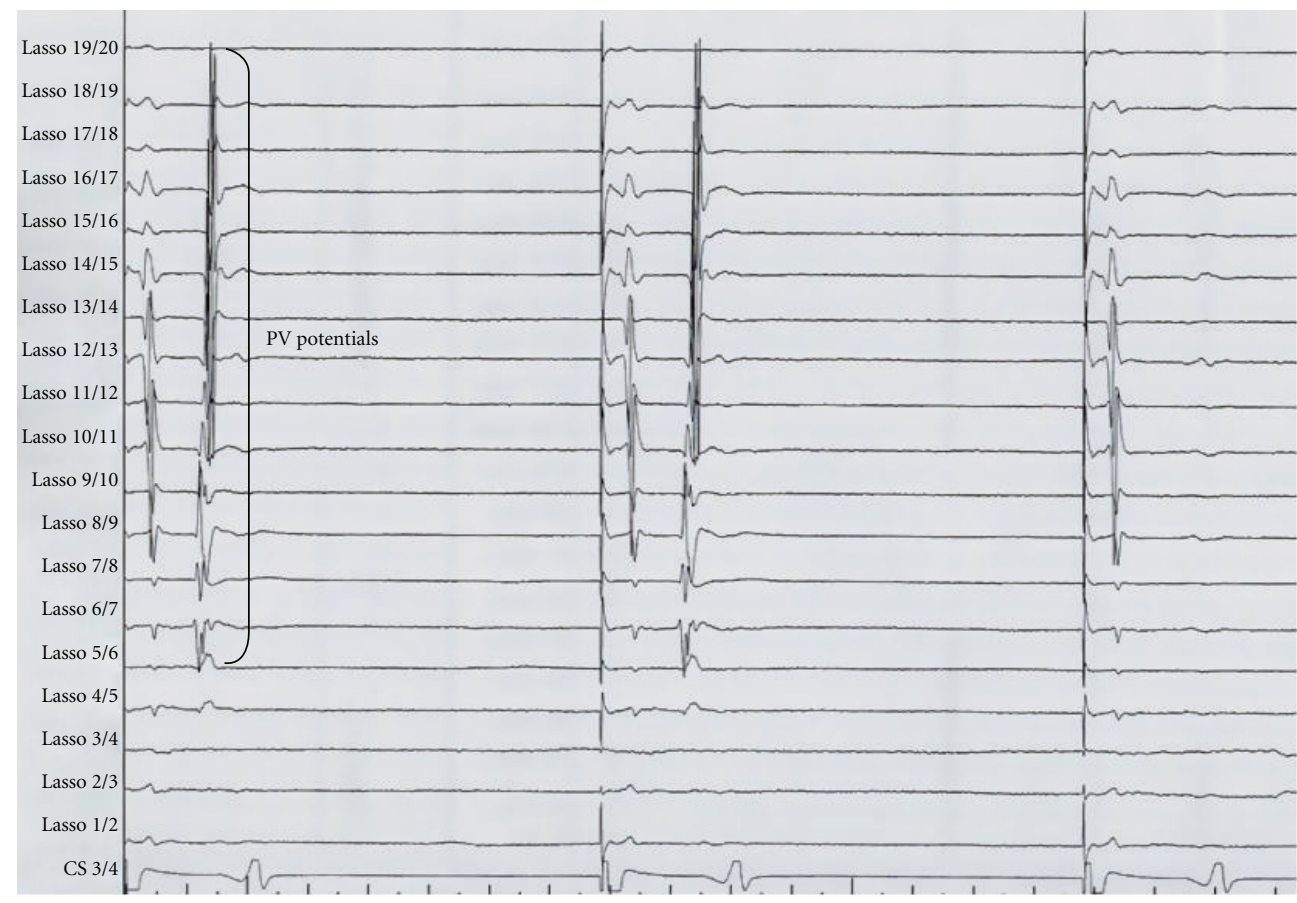

FIGURE 2: Pulmonary vein (PV) potentials recorded from the Lasso catheter bipoles during pacing from the coronary sinus (CS 3/4). In this example, the PV potentials are disappeared in the third-paced beat indicating entrance block.

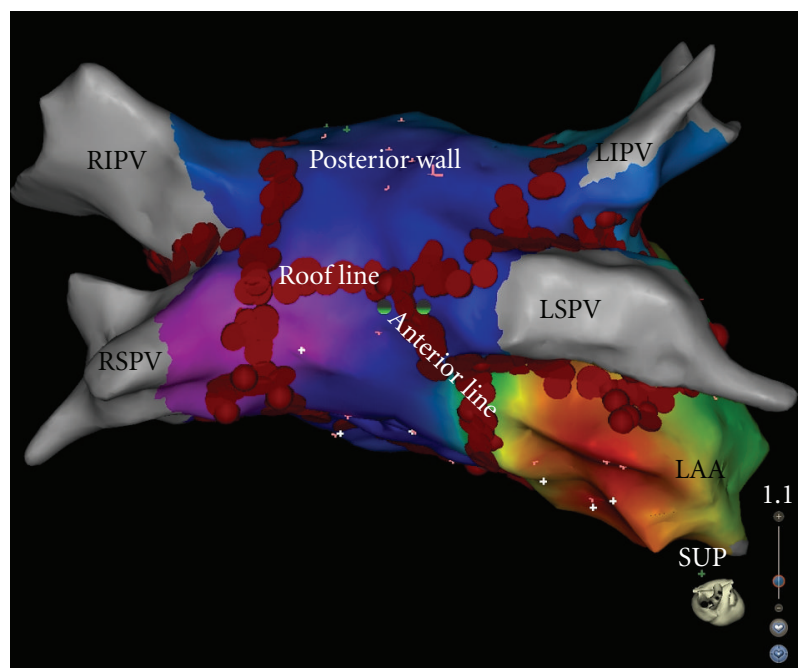

FIGURE 3: Three-dimensional reconstruction of the left atrium using the CARTO 3 map system (Biosence Webster, USA) showing large circumferential ablation lesions around both ipsilateral veins along with roof and anterior lines. LAA: left atrial appendage; LSPV: left superior pulmonary vein; LIPV: left inferior pulmonary vein; RSPV: right superior pulmonary vein; RIPV: right inferior pulmonary vein.

3.2. Linear Ablation. Based on the high success rates of surgical MAZE procedures [38], several attempts have been made to reproduce these results by percutaneous catheterbased linear ablations. Linear lesions are intended to modify the arrhythmogenic LA substrate and atrial macro-reentrant circuits involved in maintenance of $\mathrm{AF}[30,31,39$,
40]. Addition of linear lesions has been associated with conversion of AF either directly to sinus rhythm or to atrial tachycardia (AT), demonstrating that such lesions significantly modify the substrate for AF [40]. Linear lesions usually include a roof line connecting the left and right superior PVs and a mitral line connecting the mitral annulus to the left inferior PV (Figure 3) [30, 31, 39, 40]. An anterior line connecting the anterior or anterolateral mitral annulus with the left superior PV has been also proven effective for the treatment of perimitral flutter (Figure 3) [41]. Knecht et al. showed that although PVI and electrogram-based ablation without linear lesions are effective for terminating persistent AF in a significant number of patients, macro reentrant AT requiring LA linear ablation is very likely to occur during the overall follow-up period. In this study, after a followup of more than 2 years, among all the patients ablated for persistent AF, 96\% ultimately required a roofline and $86 \%$ a mitral line [40]. Linear lesions applied at the mitral isthmus or the roof of the LA increase the AF cycle length by a mean of $20 \mathrm{~ms}$ and exert a favourable impact on elimination of AF [39]. In the study of Jaïs et al., 68\% of patients required an ablation within the coronary sinus facing the endocardial aspect of the mitral isthmus because of persisting epicardial conduction [39].

LA linear ablation still remains technically challenging. Bidirectional block across the lines has to be confirmed in order to assess completeness of linear lesions [39, 40]. Conduction block of the roof line can be accomplished more frequently compared with the mitral isthmus line. When complete linear block could not be achieved during the index procedure, the incidence of subsequent roof or mitral isthmus-dependent macro-re-entrant AT is higher. In 
particular, the incidence of macro-re-entrant ATs in patients with and without conduction block of the roof line at the index procedure was $19 \%$ and $50 \%$, respectively [40]. The rates for mitral isthmus line were $26 \%$ and $56 \%$, respectively [40].

Willems et al. have investigated the effectiveness of additional substrate modification by left atrial linear lesions as compared with PVI alone in patients with persistent AF in a prospective randomized study [42]. After a mean followup time of 487 days, only $20 \%$ of patients undergoing stand alone PVI remained in sinus rhythm when compared with $69 \%$ following PVI combined with substrate modification. This study clearly shows that PVI alone is insufficient for the treatment of patients with persistent AF. In the same line, Fassini et al. confirmed the additional benefit of mitral isthmus ablation in patients with persistent AF [43]. In a systematic review, PV antral ablation or PVAI along with linear substrate modification was associated with a singleprocedure, drug-free clinical success rate ranging from $11 \%$ to $74 \%$ at approximately 1.5 years [33]. The highly variable success rate is possibly related to different procedural endpoints and/or criteria for linear lesion contiguity.

\subsection{Complex-Fractionated Atrial Electrograms. CFAEs areas} represent potential AF substrate sites and are now considered as important targets for $\mathrm{AF}$ catheter ablation $[3,4]$. CFAEs indicate sites of slow conduction, wavefront collision, conduction block, or anchor points for reentrant circuits based on the findings of an epicardial unipolar mapping study in humans [44]. CFAEs have also been proposed to indicate sites of ganglionated plexi, as shorter effective refractory period at these sites would allow higher-frequency activation [45]. CFAEs were initially defined by Nademanee et al. as (i) atrial electrograms that are fractionated and composed of two or more deflections averaged over a 10second recording period and (ii) atrial electrograms with a very short cycle length $(\leq 120 \mathrm{~ms})$ with or without multiple potentials when compared with the atrial cycle length recorded from other parts of the atria [46]. Haïssaguerre et al. performed LA ablation at all sites displaying any of the following electrogram features: continuous electrical activity, CFAEs as previously defined, sites with a gradient of activation (significant electrogram offset between the distal and proximal recording bipoles on the map electrode), or regions with a cycle length shorter than the mean left atrial appendage AF cycle length [15]. End points for ablation at sites of CFAEs include complete elimination of CFAEs or slowing and organization of local electrograms [15, 46, 47]. An important limitation of this new approach is that CFAEs are identified by visual inspection, and therefore this process is highly dependent on the operator's judgment. The lack of a consistent, reproducible technique for measuring CFAEs represents a significant challenge in the implementation of the results of studies assessing the impact of CFAEs ablation on procedural outcome. Verma et al. used an automated algorithm (Ensite NavX, St Jude Medical) to define CFAEs sites with cycle lengths $\leq 120 \mathrm{~ms}$ [48].

Nademanee et al. have initially showed that ablation of CFAEs alone resulted in termination of AF without external cardioversion in $95 \%$ of patients persistent or paroxysmal $\mathrm{AF}$ and at one-year followup, 91\% of patients were free of arrhythmia and symptoms [46]. On the contrary, Oral et al. reported a modest short-term efficacy of the ablation procedure when targeting only the CFAEs [44]. In particular, only $33 \%$ of patients were in sinus rhythm without the use of antiarrhythmic drugs after the index ablation [47]. Similarly, Estner et al. showed that ablation of CFAEs as a standalone ablation strategy seems insufficient for the treatment of patients with persistent AF [49]. CFAEs ablation alone has been associated with a single-procedure, drug-free success rates ranging from $24 \%$ to $63 \%$ at approximately 1 year [33].

A combined approach of PVI or PVAI and CFAEs ablation in persistent AF leads to acute AF termination in $66 \%$ and long-term maintenance of sinus rhythm in $74 \%$ of cases [50]. The role of ablation of CFAEs following PVAI was investigated in two recent randomized studies. Elayi et al. have shown that CFAE plus PVAI (61\%) outperformed PVAI (40\%), which was superior to PV antral ablation without electrical isolation (11\%) [35]. Oral et al. randomized 119 consecutive patients with long-standing persistent AF to PVAI or PVAI and further CFAEs ablation. These authors found that up to $2 \mathrm{~h}$ of additional ablation of CFAEs after PVAI does not appear to improve clinical outcomes in patients with long-standing persistent AF [34]. In the same line, Bencsik et al. have recently demonstrated that CFAEs ablation guided by a dedicated software algorithm and performed after PVAI had no significant impact on the fibrillatory process and displayed a minor role in achieving higher rates of termination and noninducibility in patients with persistent AF [51]. In a systematic review, the singleprocedure, drug-free success rates of CFAEs ablation in persistent and long-standing persistent $\mathrm{AF}$ as an adjunct to PV antrum ablation, PVAI or PVAI and linear ablation were $50-51 \%, 36-61 \%$, and 68\%, respectively [33]. A recent meta-analysis of six randomized trial has demonstrated that PVI followed by adjunctive CFAEs ablation is associated with increased freedom from AF after a single procedure [52]. However, CFAEs ablation significantly increased mean procedural, mean fluoroscopy, and mean radiofrequency energy application times [52].

3.4. Nonpulmonary Vein Foci. Although the PVs are a dominant source of AF, non-PV ectopic activity can trigger $\mathrm{AF}$, and ablation of these ectopic activities can eliminate AF in a specific group of patients. Non-PV triggers are more commonly observed in patients with persistent $(8.2 \%)$ and long-standing persistent AF (19.1\%) in relation to those with paroxysmal AF (2.9\%) [53]. Ablation of non-PV foci following PVI can organize persistent AF into focal or macrore-entrant atrial tachycardias, which can be eliminated, resulting in maintenance of sinus rhythm in the majority of patients $[54,55]$. The majority of non-PV foci responsible for initiation of AF are located in the superior vena cava, crista terminalis, coronary sinus, LA free wall, LA appendage, and ligament of Marshall [54, 55].

3.5. Stepwise Catheter Ablation Approach. According to the Bordeaux group, $[15,16,30-32,56,57]$ the stepwise catheter 
ablation approach for persistent AF includes (i) PVI as the initial step aiming at elimination of PV electrograms, (ii) electrogram-based ablation aiming at CFAEs and electrograms with activation gradient of at least $70 \mathrm{~ms}$ between the distal and proximal recording bipoles of the mapping catheter, (iii) if AF sustains following PVI and electrogrambased ablation, linear ablation is carried out including roof and mitral isthmus lines, (iv) finally, the right atrium (RA) and superior vena cava are targeted for ablation if implicated as a source perpetuating AF and only after all LA ablation steps. Using this sequential ablation, the Bordeaux group reported termination of chronic AF (including patients with persistent AF) by conversion to either sinus rhythm or atrial tachycardia in $87 \%$ of patients during the index procedure and freedom from AF after an 11-month follow-up period in $95 \%$ of patients $[15,16]$. Interestingly, AF was terminated in only $5 \%$ of patients by PVI, in $60 \%$ after electrogrambased ablation, and in $84 \%$ following linear ablation [15]. AF cycle length has been reported as the strongest independent predictor of procedural AF termination [57]. In a similar study, the single-procedure, drug-free success rate was 55\% [58]. Rostock et al. are the only group outside of Bordeaux to report on the stepwise procedure in patients with persistent and long-standing persistent AF. In this series, the stepwise approach was associated with a lower single-procedure, drug-free success rate of $38 \%$ at 20 months, which improved to $81 \%$ with the integration of repeat procedures [59]. New studies from different centers are required to validate the efficacy of this ablation strategy.

\section{End-Points of Catheter Ablation for Persistent and Long-Standing AF}

The goal of catheter ablation in persistent AF is trigger elimination and substrate modification. Previous studies have suggested that termination of AF during ablation in patients with persistent and long-standing AF is predictive of longterm maintenance of sinus rhythm $[15,16,34,56,57,60]$. Therefore, restoration of sinus rhythm that occurs directly or more commonly via one or more intermediate ATs may be considered as a procedural end-point. It is of paramount importance to evaluate the presence of electrical PVI as well as the integrity of linear lesions following restoration of sinus rhythm. Even though, CFAEs ablation has become "widespread" in patients with persistent and long-standing AF, important questions regarding procedural end-points still exist. The primary end-points during ablation of AF with this approach are either complete elimination of the areas with CFAEs or conversion of AF to sinus rhythm (either directly or first to an AT) $[15,46,47,56,58]$. Although the most robust end-point is AF termination, this generally requires very long procedure times.

As previously stated, restoration of sinus rhythm appears an intuitively ideal end-point. In a prospective study of 153 patients who underwent catheter ablation of persistent AF, a lower incidence of AF recurrence was demonstrated in those patients in whom AF was terminated during the index procedure compared to those without termination ( 5 versus
39\%) [56]. During ablation of persistent and long-standing AF with various strategies, the arrhythmia often organizes into a regular AT and sometimes even terminates into sinus rhythm [15]. In a recent prospective study, Elayi et al. assessed the AF termination mode during catheter ablation in 306 patients with long-lasting persistent AF and whether it predicts long-term sinus rhythm maintenance [60]. During AF ablation, only 6 out of 306 patients converted directly to sinus rhythm and 172 patients organized into AT. AF termination during ablation (conversion to sinus rhythm or AT) predicts the mode of arrhythmia recurrence (AT versus $\mathrm{AF}$ ), but did not impact on long-term sinus rhythm maintenance after a single or two procedures [60].

In clinical practice, the ideal end-point for AF catheter ablation is freedom from AF without the use of antiarrhythmic medications. There is much controversy regarding the monitoring period as well as the minimum acceptable AF burden. Current definitions of freedom from AF include absence of AF, AF episodes lasting up to $30 \mathrm{~s}$, and absence of symptomatic AF $[3,4]$. A "blanking period" of up to 3 months after ablation, during which antiarrhythmic medications may be continued and direct current cardioversion can be performed for early recurrences of arrhythmias, appears to be adequate in order to access the efficacy of an ablation strategy $[3,4]$.

\section{Which Is the Price for an Extensive Ablation Procedure in the Setting of Persistent AF?}

Longer procedure time, longer fluoroscopy time, higher complication rates, and high rates of postprocedural ATs are the main consequences of an extensive ablation procedure carried out in the setting of persistent and long-standing persistent AF. In a systematic review of 22 studies (1690 patients with persistent and long-lasting persistent $\mathrm{AF}$ ), complications have been reported in $4.4 \%$ and included pericardial tamponade/effusion (1.4\%), vascular complications $(0.80 \%)$, symptomatic pulmonary vein stenosis $(0.71 \%)$, cerebrovascular events $(0.65 \%)$, phrenic nerve injuries $(0.3 \%)$, and atrioesophageal fistula $(0.06 \%)$ [33]. Weber et al. have also reported the development of pulmonary edema following AF ablation, possibly in the setting of a systemic inflammatory response syndrome [61]. Cardiac tamponade is by far the most common fatal complication of AF ablation, occurring in $0.8-6 \%[3,4,62-67]$. Death is an uncommon complication of AF ablation, occurring in $0.1-0.15 \%$ of subjects [3, 4, 62-67]. Operator's experience is extremely important regarding safety issues of catheter ablation of AF. Spragg et al. reported that complication rates were higher during the first 100 cases $(9.0 \%)$ than during the subsequent $541(4.3 \%)$ [67]. Table 1 summarizes the most common complications occurring during catheter ablation of AF.

ATs following the index procedure can occur intraprocedurally or postprocedurally (early or delayed) [68]. Using a stepwise ablation strategy, Haissaguerre et al. demonstrated that persistent AF is usually terminated to an AT (86.5\%) (focal or macro-re-entry) and less frequently directly to sinus rhythm (13.5\%) [15]. This conversion is preceded by 
TABLE 1: Complications during catheter ablation of persistent and long-standing persistent AF.

\begin{tabular}{lll}
\hline Type of complication & Symptoms & Incidence \\
\hline Atrio-oesophageal fistula & Fever, dysphagia & Rare $(0.06 \%)$ \\
$\begin{array}{l}\text { Vascular complications (Arteriovenous fistula, arterial aneurysm, } \\
\text { haematoma) }\end{array}$ & Pain at puncture site & $0.8 \%$ \\
Phrenic nerve injury & Cough, dyspnea, atelectasis, and/or thoracic pain & $0.3 \%$ \\
Pulmonary vein stenosis & $\begin{array}{l}\text { Cough, hemoptysis, dyspnea, chest pain, and } \\
\text { recurrent lung infections }\end{array}$ & $0.71 \%$ \\
Pulmonary edema (18-48 h after the procedure) & Dyspnea, fever, elevated CRP levels & $0.65 \%$ \\
Cerebrovascular events & Neurological deficit & $1.4 \%$ \\
Tambonade/Effusion & Hypotension, dyspnea cardiac arrest & $(0.8-6 \%)$ \\
Death & & $0.1-0.15 \%$ \\
\hline
\end{tabular}

prolongation of fibrillatory cycle length, with the greatest magnitude occurring during ablation at the anterior LA, coronary sinus, and PV-LA junction [15]. These intraprocedural ATs are more commonly macro-re-entrant circuits involving the mitral or cavotricuspid isthmus or LA roof $[68,69]$. Postprocedural (secondary) ATs are often incessant and very poorly tolerated by the patient. Postprocedural ATs are a rapidly growing clinical problem due to the expansion of the ablation strategies in the setting of persistent $\mathrm{AF}$ $[3,4]$. The incidence of these arrhythmias is higher following circumferential PV ablation than after segmental PVI and is even higher when linear ablation is performed within the LA $[68,69]$. ATs have been shown to occur in about $40 \%$ of patients who undergo the stepwise ablation approach $[15,69,70]$. These ATs can be focal or macro-re-entrant. More recently, a third intermediate AT category called "localized re-entry" has been reported $[69,70]$. Focal ATs are defined by centrifugal activation from a localized region. If there is cycle length variation in left atrial appendage and coronary sinus $>15 \%$, focal mechanism is the most likely diagnosis. However, variation $<15 \%$ does not rule out focal mechanism [70]. A macro-re-entrant mechanism is defined by demonstrating the entire cycle length of activity in a chamber with entrainment at $\geq 2$ sites displaying a post-pacing interval of $<20 \mathrm{msec}$ longer than the tachycardia cycle length [70]. Most macro-re-entrant postprocedural ATs arise from a proarrhythmic effect of incomplete conduction block of linear lesions [68-70]. However, Knecht et al. showed that these macro-re-entrant circuits may also occur in the majority of patients without previously performed linear lesions in the long-term followup [40]. PV recovery should be also considered as a cause of AF recurrence, but may also contribute to either re-entrant or focal ATs [68-70]. Management of secondary ATs includes effective anticoagulation minimizing the risk of thromboembolic events, prevention of tachycardia-induced cardiomyopathy, and control of symptoms. One-third of patients who develop ATs in the first few weeks after AF ablation may not develop recurrent atrial arrhythmias during followup [69]. An initial conservative management strategy including rate control and cardioversion seems reasonable $[68,69]$. Symptomatic patients with ATs that persist beyond 2-3 months should be offered catheter ablation [69].

\section{Conclusions}

In conclusion, catheter ablation of persistent and longstanding persistent AF remains challenging for the electrophysiologists. Up to now, no single strategy is uniformly effective in patients with persistent and long-standing AF. The risk/benefit ratio of an extensive ablation approach has to be carefully evaluated. More lesions prolong not only procedure and fluoroscopy times, but also increase the risk of complications including ATs. For this purpose, the longterm success rates of certain ablation strategies need to be evaluated in randomized trials.

\section{References}

[1] W. B. Kannel, R. D. Abbott, D. D. Savage, and P. M. McNamara, "Epidemiologic features of chronic atrial fibrillation. The Framingham study," New England Journal of Medicine, vol. 306, no. 17, pp. 1018-1022, 1982.

[2] The AFFIRM Investigators, "Relationships between Sinus Rhythm, treatment, and survival in the atrial fibrillation follow-up investigation of rhythm management (AFFIRM) study," Circulation, vol. 109, no. 12, pp. 1509-1513, 2004.

[3] H. Calkins, J. Brugada, D. L. Packer et al., "HRS/EHRA/ECAS expert consensus statement on catheter and surgical ablation of atrial fibrillation: recommendations for personnel, policy, procedures and follow-up. A report of the Heart Rhythm Society (HRS) Task Force on catheter and surgical ablation of atrial fibrillation," Europace, vol. 9, no. 6, pp. 335-379, 2007.

[4] A. Natale, A. Raviele, T. Arentz et al., "Venice chart international consensus document on atrial fibrillation ablation," Journal of Cardiovascular Electrophysiology, vol. 18, no. 5, pp. 560-580, 2007.

[5] A. J. Camm, P. Kirchhof, G. Y. Lip et al., "Guidelines for the management of atrial fibrillation: the Task Force for the Management of Atrial Fibrillation of the European Society of Cardiology (ESC)," Europace, vol. 12, pp. 1360-1420, 2010.

[6] C. Pappone, S. Rosanio, G. Augello et al., "Mortality, morbidity, and quality of life after circumferential pulmonary vein 
ablation for atrial fibrillation: outcomes from a controlled nonrandomized long-term study," Journal of the American College of Cardiology, vol. 42, no. 2, pp. 185-197, 2003.

[7] D. J. Wilber, C. Pappone, P. Neuzil et al., "Comparison of antiarrhythmic drug therapy and radiofrequency catheter ablation in patients with paroxysmal atrial fibrillation: a randomized controlled trial," Journal of the American Medical Association, vol. 303, no. 4, pp. 333-340, 2010.

[8] A. Noheria, A. Kumar, J. V. Wylie, and M. E. Josephson, "Catheter ablation vs antiarrhythmic drug therapy for atrial fibrillation: a systematic review," Archives of Internal Medicine, vol. 168, no. 6, pp. 581-586, 2008.

[9] J. P. Piccini, R. D. Lopes, M. H. Kong, V. Hasselblad, K. Jackson, and S. M. Al-Khatib, "Pulmonary vein isolation for the maintenance of sinus rhythm in patients with atrial fibrillation a meta-analysis of randomized, controlled trials," Circulation Arrhythmia and Electrophysiology, vol. 2, no. 6, pp. 626-633, 2009.

[10] P. Jaïs, B. Cauchemez, L. Macle et al., "Catheter ablation versus antiarrhythmic drugs for atrial fibrillation: the A4 study," Circulation, vol. 118, no. 24, pp. 2498-2505, 2008.

[11] G. B. Forleo, M. Mantica, L. De Luca et al., "Catheter ablation of atrial fibrillation in patients with diabetes mellitus type 2: results from a randomized study comparing pulmonary vein isolation versus antiarrhythmic drug therapy," Journal of Cardiovascular Electrophysiology, vol. 20, no. 1, pp. 22-28, 2009.

[12] M. Haïssaguerre, P. Jaïs, D. C. Shah et al., "Spontaneous initiation of atrial fibrillation by ectopic beats originating in the pulmonary veins," New England Journal of Medicine, vol. 339, no. 10, pp. 659-666, 1998.

[13] S. K. Mainigi, W. H. Sauer, J. M. Cooper et al., "Incidence and predictors of very late recurrence of atrial fibrillation after ablation," Journal of Cardiovascular Electrophysiology, vol. 18, no. 1, pp. 69-74, 2007.

[14] M. Hocini, P. Sanders, P. Jaïs et al., "Techniques for curative treatment of atrial fibrillation," Journal of Cardiovascular Electrophysiology, vol. 15, no. 12, pp. 1467-1471, 2004.

[15] M. Haïssaguerre, P. Sanders, M. Hocini et al., "Catheter ablation of long-lasting persistent atrial fibrillation: critical structures for termination," Journal of Cardiovascular Electrophysiology, vol. 16, no. 11, pp. 1125-1137, 2005.

[16] M. Haïssaguerre, M. Hocini, P. Sanders et al., "Catheter ablation of long-lasting persistent atrial fibrillation: clinical outcome and mechanisms of subsequent arrhythmias," Journal of Cardiovascular Electrophysiology, vol. 16, no. 11, pp. 1138-1147, 2005.

[17] I. Savelieva and J. Camm, "Update on atrial fibrillation: part I," Clinical Cardiology, vol. 31, no. 2, pp. 55-62, 2008.

[18] S. A. Chen, M. H. Hsieh, C. T. Tai et al., "Initiation of atrial fibrillation by ectopic beats originating from the pulmonary veins: electrophysiological characteristics, pharmacological responses, and effects of radiofrequency ablation," Circulation, vol. 100, no. 18, pp. 1879-1886, 1999.

[19] G. K. Moe and J. A. Abildskov, "Atrial fibrillation as a self-sustaining arrhythmia independent of focal discharge," American Heart Journal, vol. 58, no. 1, pp. 59-70, 1959.

[20] M. A. Allessie, W. J. E. P. Lammers, F. I. M. Bonke, and J. Hollen, "Experimental evaluation of Moe's multiple wavelet hypothesis of atrial fibrillation," in Cardiac Electrophysiology and Arrhythmias, D. P. Zipes and J. Jalife, Eds., pp. 265-275, Grune \& Stratton, NewYork, NY, USA, 1985.

[21] K. T. S. Konings, C. J. H. J. Kirchhof, J. R. L. M. Smeets, H. J. J. Wellens, O. C. Penn, and M. A. Allessie, "High-density mapping of electrically induced atrial fibrillation in humans," Circulation, vol. 89, no. 4, pp. 1665-1680, 1994.

[22] M. C. E. F. Wijffels, C. J. H. J. Kirchhof, R. Dorland, and M. A. Allessie, "Atrial fibrillation begets atrial fibrillation: a study in awake chronically instrumented goats," Circulation, vol. 92, no. 7, pp. 1954-1968, 1995.

[23] H. Oral, B. P. Knight, H. Tada et al., "Pulmonary vein isolation for paroxysmal and persistent atrial fibrillation," Circulation, vol. 105, no. 9, pp. 1077-1081, 2002.

[24] H. Oral, B. P. Knight, M. Özaydin et al., "Segmental ostial ablation to isolate the pulmonary veins during atrial fibrillation: feasibility and mechanistic insights," Circulation, vol. 106, no. 10, pp. 1256-1262, 2002.

[25] C. Pappone, S. Rosanio, G. Oreto et al., "Circumferential radiofrequency ablation of pulmonary vein ostia: a new anatomic approach for curing atrial fibrillation," Circulation, vol. 102, no. 21, pp. 2619-2628, 2000.

[26] T. Arentz, R. Weber, G. Bürkle et al., "Small or large isolation areas around the pulmonary veins for the treatment of atrial fibrillation? Results from a prospective randomized study," Circulation, vol. 115, no. 24, pp. 3057-3063, 2007.

[27] F. Ouyang, D. Bänsch, S. Ernst et al., "Complete isolation of left atrium surrounding the pulmonary veins: new insights from the double-lasso technique in paroxysmal atrial fibrillation," Circulation, vol. 110, no. 15, pp. 2090-2096, 2004.

[28] H. Ghanbari, M. Schmidt, C. Machado, N. M. Segerson, and M. Daccarett, "Ablation strategies for atrial fibrillation," Expert Review of Cardiovascular Therapy, vol. 7, no. 9, pp. 1091-1101, 2009.

[29] M. Wright, M. Haïssaguerre, S. Knecht et al., "State of the art: catheter ablation of atrial fibrillation," Journal of Cardiovascular Electrophysiology, vol. 19, no. 6, pp. 583-592, 2008.

[30] M. P. Smelley and B. P. Knight, "Approaches to catheter ablation of persistent atrial fibrillation," Heart Rhythm, vol. 6, no. 8, pp. S33-S38, 2009.

[31] K. T. Lim, S. Matsuo, M. D. O’Neill et al., “Catheter ablation of persistent and permanent atrial fibrillation: bordeaux experience," Expert Review of Cardiovascular Therapy, vol. 5, no. 4, pp. 655-662, 2007.

[32] H. Oral, "Catheter ablation for chronic atrial fibrillation," Heart Rhythm, vol. 4, no. 5, pp. 691-694, 2007.

[33] A. G. Brooks, M. K. Stiles, J. Laborderie et al., "Outcomes of long-standing persistent atrial fibrillation ablation: a systematic review," Heart Rhythm, vol. 7, no. 6, pp. 835-846, 2010.

[34] H. Oral, A. Chugh, K. Yoshida et al., "A randomized assessment of the incremental role of ablation of complex fractionated atrial electrograms after antral pulmonary vein isolation for long-lasting persistent atrial fibrillation," Journal of the American College of Cardiology, vol. 53, no. 9, pp. 782789, 2009.

[35] C. S. Elayi, A. Verma, L. Di Biase et al., "Ablation for longstanding permanent atrial fibrillation: results from a randomized study comparing three different strategies," Heart Rhythm, vol. 5, no. 12, pp. 1658-1664, 2008.

[36] R. R. Tilz, K. R. J. Chun, B. Schmidt et al., "Catheter ablation of long-standing persistent atrial fibrillation: a lesson from circumferential pulmonary vein isolation," Journal of Cardiovascular Electrophysiology, vol. 21, no. 10, pp. 10851093, 2010.

[37] A. Cheema, J. Dong, D. Dalal et al., "Circumferential ablation with pulmonary vein isolation in permanent atrial fibrillation," American Journal of Cardiology, vol. 99, no. 10, pp. 1425$1428,2007$. 
[38] J. L. Cox, R. B. Schuessler, H. J. D’Agostino et al., “The surgical treatment of atrial fibrillation: III. Development of a definitive surgical procedure," Journal of Thoracic and Cardiovascular Surgery, vol. 101, no. 4, pp. 569-583, 1991.

[39] P. Jaïs, M. Hocini, M. D. O'Neill et al., "How to perform linear lesions," Heart Rhythm, vol. 4, no. 6, pp. 803-809, 2007.

[40] S. Knecht, M. Hocini, M. Wright et al., "Left atrial linear lesions are required for successful treatment of persistent atrial fibrillation," European Heart Journal, vol. 29, no. 19, pp. 23592366, 2008.

[41] S. Tzeis, A. Luik, C. Jilek et al., "The modified anterior line: an alternative linear lesion in perimitral flutter," Journal of Cardiovascular Electrophysiology, vol. 21, no. 6, pp. 665-670, 2010.

[42] S. Willems, H. Klemm, T. Rostock et al., "Substrate modification combined with pulmonary vein isolation improves outcome of catheter ablation in patients with persistent atrial fibrillation: a prospective randomized comparison," European Heart Journal, vol. 27, no. 23, pp. 2871-2878, 2006.

[43] G. Fassini, S. Riva, R. Chiodelli et al., "Left mitral isthmus ablation associated with PV isolation: long-term results of a prospective randomized study," Journal of Cardiovascular Electrophysiology, vol. 16, no. 11, pp. 1150-1156, 2005.

[44] K. T. S. Konings, J. L. R. M. Smeets, O. C. Penn, H. J. J. Wellens, and M. A. Allessie, "Configuration of unipolar atrial electrograms during electrically induced atrial fibrillation in humans," Circulation, vol. 95, no. 5, pp. 1231-1241, 1997.

[45] J. Lin, B. J. Scherlag, J. Zhou et al., "Autonomic mechanism to explain complex fractionated atrial electrograms (CFAE)," Journal of Cardiovascular Electrophysiology, vol. 18, no. 11, pp. 1197-1205, 2007.

[46] K. Nademanee, J. McKenzie, E. Kosar et al., "A new approach for catheter ablation of atrial fibrillation: mapping of the electrophysiologic substrate," Journal of the American College of Cardiology, vol. 43, no. 11, pp. 2044-2053, 2004.

[47] H. Oral, A. Chugh, E. Good et al., "Radiofrequency catheter ablation of chronic atrial fibrillation guided by complex electrograms," Circulation, vol. 115, no. 20, pp. 2606-2612, 2007.

[48] A. Verma, R. Mantovan, L. MacLe et al., "Substrate and Trigger Ablation for Reduction of Atrial Fibrillation (STAR AF): a randomized, multicentre, international trial," European Heart Journal, vol. 31, no. 11, pp. 1344-1356, 2010.

[49] H. L. Estner, G. Hessling, G. Ndrepepa et al., "Electrogramguided substrate ablation with or without pulmonary vein isolation in patients with persistent atrial fibrillation," Europace, vol. 10, no. 11, pp. 1281-1287, 2008.

[50] H. L. Estner, G. Hessling, G. Ndrepepa et al., "Acute effects and long-term outcome of pulmonary vein isolation in combination with electrogram-guided substrate ablation for persistent atrial fibrillation," American Journal of Cardiology, vol. 101, no. 3, pp. 332-337, 2008.

[51] G. Bencsik, M. Martinek, S. Hassanein, J. Aichinger, H. J. Nesser, and H. Purerfellner, "Acute effects of complex fractionated atrial electrogram ablation on dominant frequency and regulatory index for the fibrillatory process," Europace, vol. 11, no. 8, pp. 1011-1017, 2009.

[52] M. H. Kong, J. P. Piccini, and T. D. Bahnson, "Efficacy of adjunctive ablation of complex fractionated atrial electrograms and pulmonary vein isolation for the treatment of atrial fibrillation: a meta-analysis of randomized controlled trials," Europace, vol. 13, no. 2, pp. 193-204, 2011.

[53] M. Bhargava, L. Di Biase, P. Mohanty et al., "Impact of type of atrial fibrillation and repeat catheter ablation on long-term freedom from atrial fibrillation: results from a multicenter study," Heart Rhythm, vol. 6, no. 10, pp. 1403-1412, 2009.

[54] M. Haïssaguerre, M. Hocini, P. Sanders et al., "Localized sources maintaining atrial fibrillation organized by prior ablation," Circulation, vol. 113, no. 5, pp. 616-625, 2006.

[55] S. Higa, C. T. Tai, and S. A. Chen, "Catheter ablation of atrial fibrillation originating from extrapulmonary vein areas: taipei approach," Heart Rhythm, vol. 3, no. 11, pp. 1386-1390, 2006.

[56] M. D. O’Neill, M. Wright, S. Knecht et al., "Long-term followup of persistent atrial fibrillation ablation using termination as a procedural endpoint," European Heart Journal, vol. 30, no. 9, pp. 1105-1112, 2009.

[57] S. Matsuo, N. Lellouche, M. Wright et al., "Clinical predictors of termination and clinical outcome of catheter ablation for persistent atrial fibrillation," Journal of the American College of Cardiology, vol. 54, no. 9, pp. 788-795, 2009.

[58] Y. Takahashi, M. D. O’Neill, M. Hocini et al., "Characterization of electrograms associated with termination of chronic atrial fibrillation by catheter ablation," Journal of the American College of Cardiology, vol. 51, no. 10, pp. 1003-1010, 2008.

[59] T. Rostock, D. Steven, B. Hoffmann et al., "Chronic atrial fibrillation is a biatrial arrhythmia: data from catheter ablation of chronic atrial fibrillation aiming arrhythmia termination using a sequential ablation approach," Circulation Arrhythmia and Electrophysiology, vol. 1, no. 5, pp. 344-353, 2008.

[60] C. S. Elayi, L. Di Biase, C. Barrett et al., "Atrial fibrillation termination as a procedural endpoint during ablation in longstanding persistent atrial fibrillation," Heart Rhythm, vol. 7, pp. 1216-1223, 2010.

[61] R. Weber, J. Minners, C. Restle et al., "Pulmonary edema after extensive radiofrequency ablation for atrial fibrillation," Journal of Cardiovascular Electrophysiology, vol. 19, no. 7, pp. 748-752, 2008.

[62] R. Cappato, H. Calkins, S. A. Chen et al., "Updated worldwide survey on the methods, efficacy, and safety of catheter ablation for human atrial fibrillation," Circulation Arrhythmia and Electrophysiology, vol. 3, no. 1, pp. 32-38, 2010.

[63] R. Cappato, H. Calkins, S. A. Chen et al., "Prevalence and causes of fatal outcome in catheter ablation of atrial fibrillation," Journal of the American College of Cardiology, vol. 53, no. 19, pp. 1798-1803, 2009.

[64] A. Takahashi, T. Kuwahara, and Y. Takahashi, "Complications in the catheter ablation of atrial fibrillation-incidence and management," Circulation Journal, vol. 73, no. 2, pp. 221-226, 2009.

[65] N. Dagres, G. Hindricks, H. Kottkamp et al., "Complications of atrial fibrillation ablation in a high-volume center in 1,000 procedures: still cause for concern?" Journal of Cardiovascular Electrophysiology, vol. 20, no. 9, pp. 1014-1019, 2009.

[66] E. Bertaglia, F. Zoppo, C. Tondo et al., "Early complications of pulmonary vein catheter ablation for atrial fibrillation: a multicenter prospective registry on procedural safety," Heart Rhythm, vol. 4, no. 10, pp. 1265-1271, 2007.

[67] D. D. Spragg, D. Dalal, A. Cheema et al., "Complications of catheter ablation for atrial fibrillation: incidence and predictors," Journal of Cardiovascular Electrophysiology, vol. 19, no. 6, pp. 627-631, 2008.

[68] A. J. Shah, A. Jadidi, X. Liu et al., "Atrial tachycardias arising from ablation of atrial fibrillation: a proarrhythmic bump or an antiarrhythmic turn?" Cardiology Research and Practice, vol. 2010, Article ID 950763, 2010.

[69] G. D. Veenhuyzen, S. Knecht, M. D. O’Neill et al., “Atrial tachycardias encountered during and after catheter ablation 
for atrial fibrillation. Part I: classification, incidence, management," Pacing and Clinical Electrophysiology, vol. 32, no. 3, pp. 393-398, 2009.

[70] P. Jaïs, S. Matsuo, S. Knecht et al., "A deductive mapping strategy for atrial tachycardia following atrial fibrillation ablation: importance of localized reentry," Journal of Cardiovascular Electrophysiology, vol. 20, no. 5, pp. 480-491, 2009. 


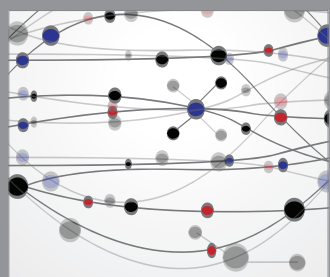

The Scientific World Journal
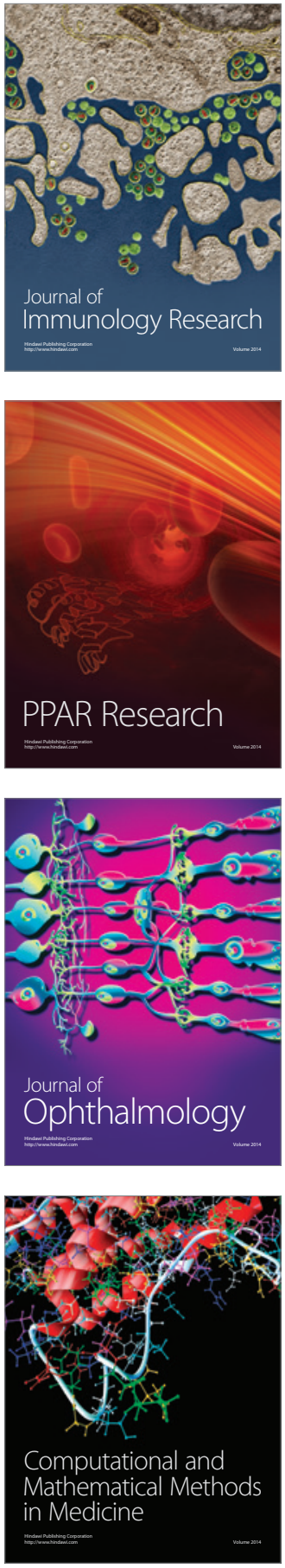

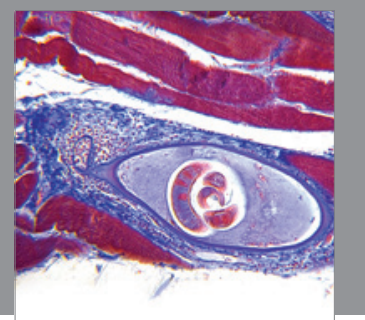

Gastroenterology

Research and Practice
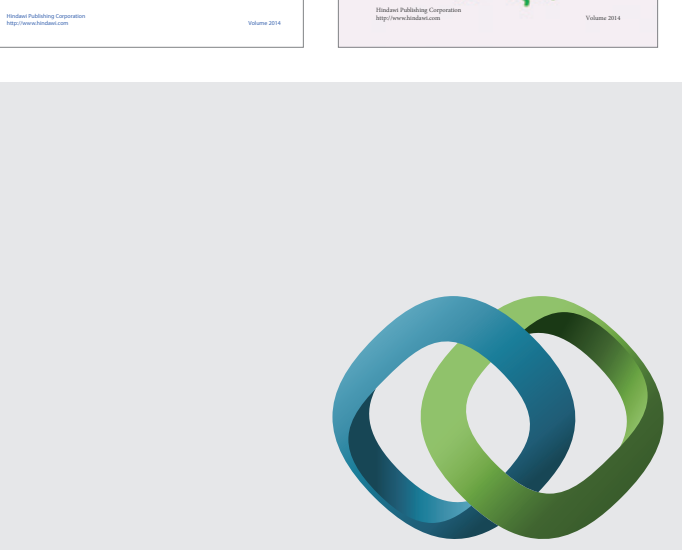

\section{Hindawi}

Submit your manuscripts at

http://www.hindawi.com
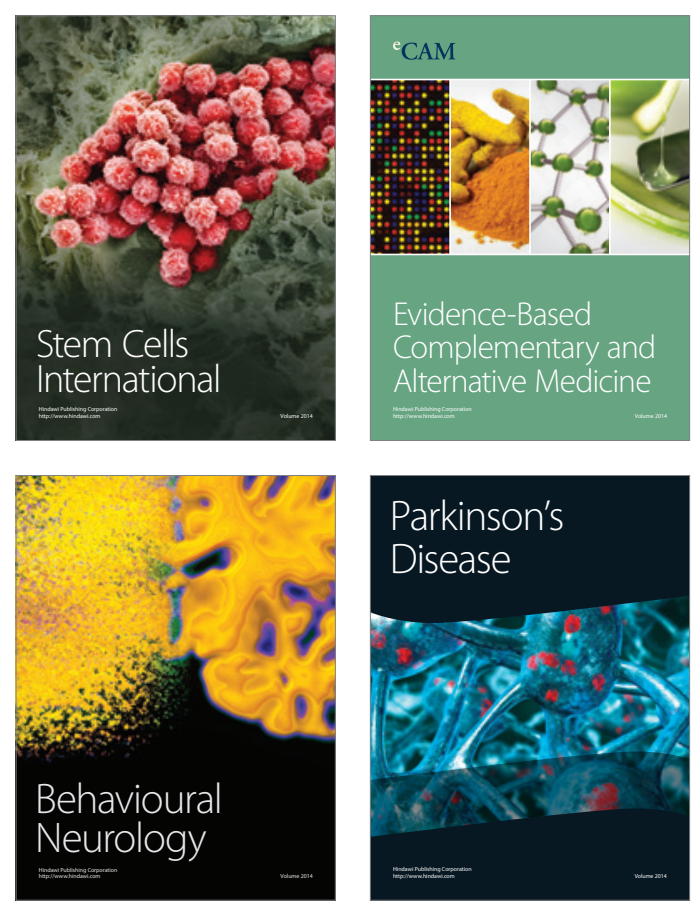

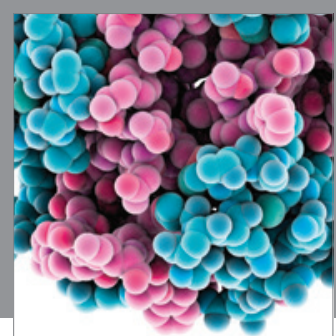

Journal of
Diabetes Research

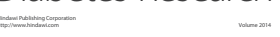

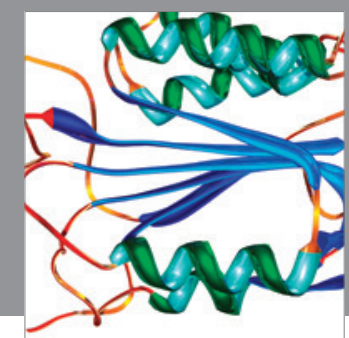

Disease Markers
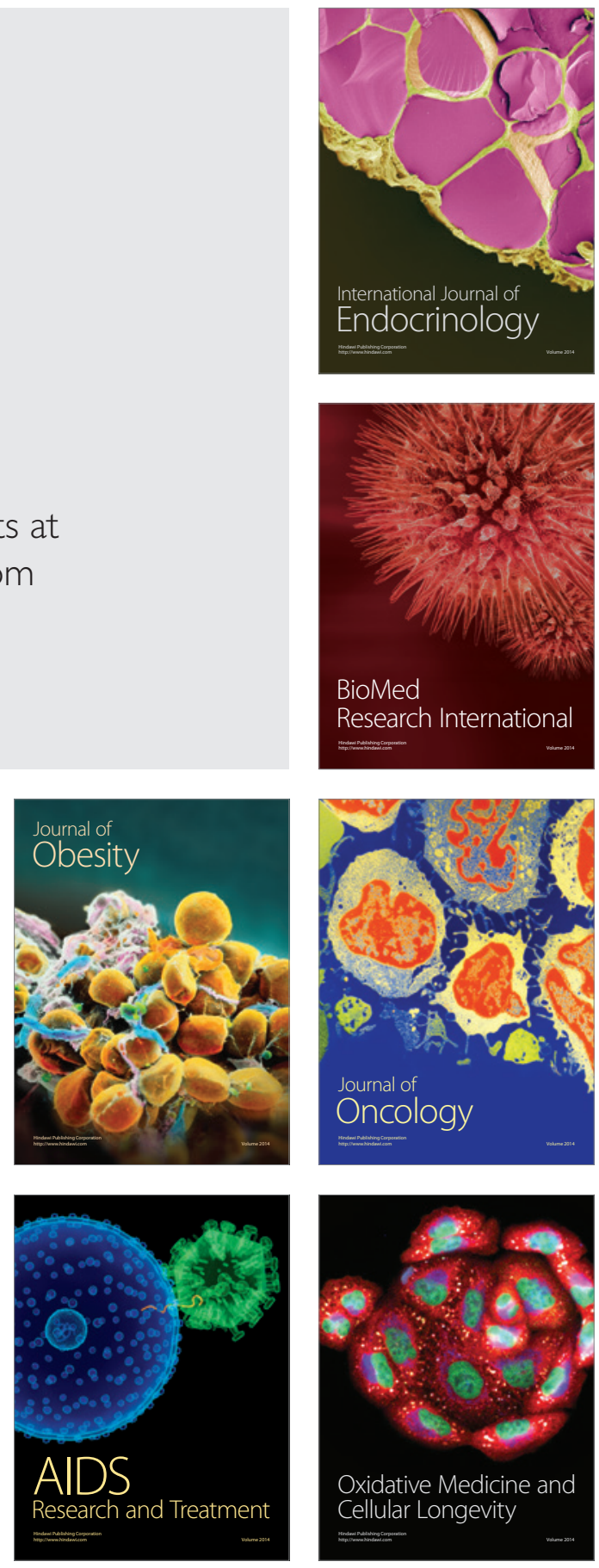